\title{
Near-Bulk Conductivity of Gold Nanowires as Nanoscale Interconnects and the Role of Atomically Smooth Interface
}

\author{
By Kevin Critchley, Bishnu P. Khanal, Marcin Ł. Górzny, Leonid Vigderman, \\ Stephen D. Evans, Eugene R. Zubarev,* and Nicholas A. Kotov**
}

The electronics industry has consistently decreased the dimensions of structural components and they are now well into the nanoscale range. Naturally, a significant portion of the chip is composed of interconnects. Besides, the engineering problems associated with short wavelength lithography to achieve smaller components, the performance of increasing number of interconnections has become one of the biggest limiting factors in device performance. ${ }^{[1,2]}$ The power loss, signal degradation, interconnection delays, and other performance limitations related to interconnects should be minimized. The importance of such a task can be seen from the perspective of power dissipation by computation elements. The energy dissipation density in electronic chips approaches that in nuclear reactors. ${ }^{[3,4]}$ Therefore, the decrease of the resistivity of metal interconnects is one of the key challenges in the design of nanoscale electronic circuits.

Intense studies on methods of preparation of interconnects by advanced lithographic techniques including e-beam lithography lead to the preparation of $\mathrm{Au}, \mathrm{Pd}, \mathrm{Pt}$, and $\mathrm{Cu}$ nanowires (NWs) with resistivities much greater than the bulk metal value (see Supporting Information). ${ }^{[5-11]}$ The reason for the drastic increase in resistivity for NWs is that charge carriers experience grain boundaries reflections and surface scattering. The smaller the diameter of the conductor, the greater this effect becomes. Even NWs with diameters much above the nanometer scale can exhibit resistivities as high as two orders of magnitude greater that the bulk. ${ }^{[12]}$

Potentially, connecting elements can be obtained from NWs synthesized in templates or in solution since lithographic substrates and the process itself can result in appearance of

[*] Prof. N. A. Kotov, Dr. K. Critchley

Department of Chemical Engineering

University of Michigan

Ann Arbor, 48109 (USA)

E-mail: kotov@umich.edu

Prof. E. R. Zubarev, Dr. B. P. Khanal, L. Vigderman

Department of Chemistry

Rice University

Houston, TX 77005 (USA)

E-mail: zubarev@rice.edu

M. Ł. Górzny, Prof. S. D. Evans

School of Physics

University of Leeds

Leeds. LS2 9JT (UK)

DOI: 10.1002/adma.201000236 multiple grain boundaries. NWs dispersed in solutions can be aligned onto substrates using several methodologies including, dielectrophoretic, ${ }^{[13-15]}$ Langmuir-Blodgett, ${ }^{[16]}$ microcontact stamping, ${ }^{[17-19]}$ molecular surface patterning, ${ }^{[20,21]}$ magnetic fields, ${ }^{[22]}$ and fluidic flow alignment techniques. ${ }^{[23,24]}$ The resistivity of NWs synthesized in templates did not improve the situation as compared to lithographical methods. The NWs of $\mathrm{Pt}, \mathrm{Au}$, and $\mathrm{Cu}$ were shown to have resistivities as high as 3, 7, 26 times of the corresponding bulk metal, respectively. ${ }^{[10,11,25]}$ Twoand four-probe transport measurements on Au and Pt NWs made by axial solution growth ${ }^{[26-28]}$ displayed resistivities from $10^{[28]}$ to as high as 100 times $^{[26,27]}$ higher than the bulk.

These observations raise several basic questions related to nanoscale interconnects. What is the theoretical limit of resistivity for monocrystalline NWs for a given diameter? Is the required near-bulk conductivity possible for NWs of very small diameters? In this study, we tried to answer these questions by making exceptionally long pentahedrally twinned Au NWs and carried out transport measurements using a multi-probe scanning tunneling microscope (STM) technique. ${ }^{[29]}$ The conductivity of these NWs was analyzed with respect to the bulk metal. Application of a theoretical approach allows one to identify important physical properties that metallic interconnects require for achieving low resistivity.

Preparation of NWs suitable for the conductivity studies represents several challenges. The solution techniques of axial growth of metal $\mathrm{NWS}^{[30-33]}$ does not result in the wires with aspect ratio sufficiently high to reliably measure the conductivity. It was possible to fabricate rod-like structures with an aspect ratio close to $20-25$ by using Au nanoparticles by three-step seed mediating method, however, this is not sufficient to accommodate available four probe methods giving the best electrical transport data. The template growth of Au NWs in porous membranes has proven to be very successful and high aspect ratios can be achieved. ${ }^{[34,35]}$ However, the resulting NWs faithfully replicate the imperfections of the template, which may enhance the scattering of charge carriers.

We found that the average length of rods in the seed-mediated method can be increased continuously when additional amount of growth solution containing $\mathrm{Au}$ (I) and ascorbic acid is added (Supporting Information). The average length of the first batch of NWs increased from ca. 300 to $2300 \mathrm{~nm}$ (standard deviation $14.6 \%$ ) whereas the diameter increased only from 22 to $29 \mathrm{~nm}$ (Fig. 1A-C). NWs with diameters of $92 \mathrm{~nm}$ (Fig. 1D) and $185 \mathrm{~nm}$ (Fig. 1E) were also synthesized by following the same procedure. The resulting $\mathrm{Au}$ NWs undergo slow gravitational precipitation 

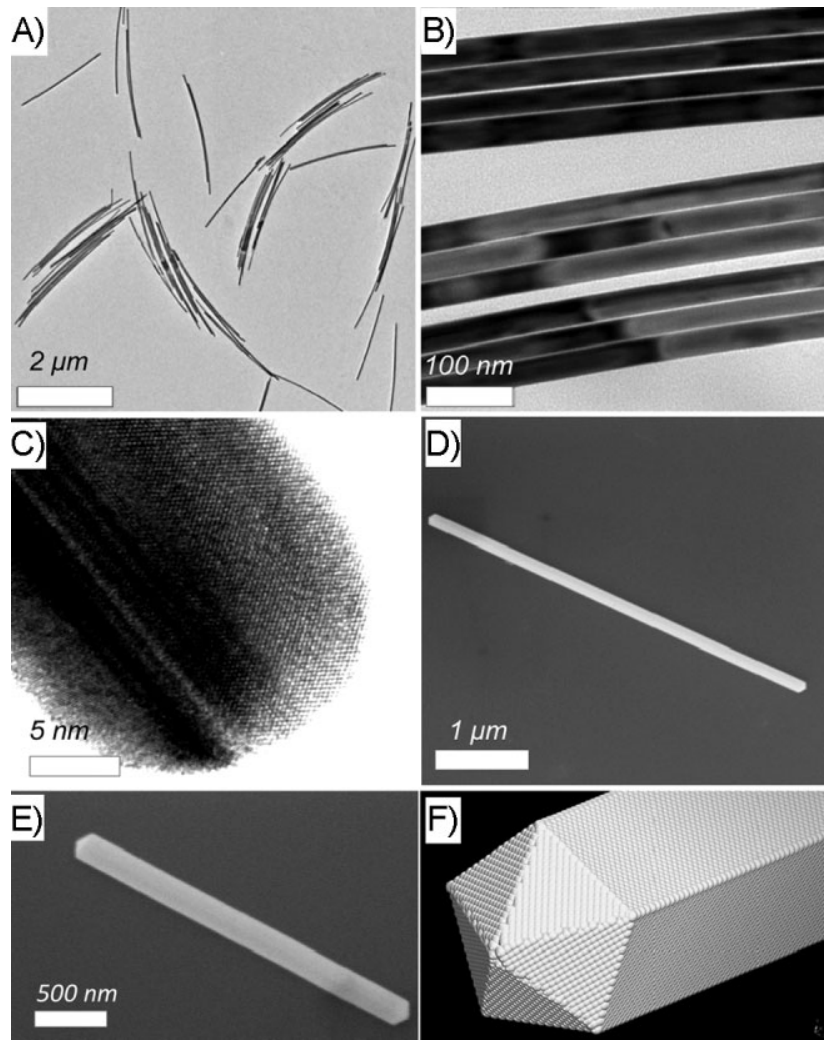

Figure 1. Low- (A) and high-magnification (B) transmission electron microscopy (TEM) images of 29-nm-diameter Au NWs stabilized by CTAB High-resolution (C) TEM image of the $29 \mathrm{~nm}$ diameter pentahedrally twinned $\mathrm{Au} N$ NS showing the crystalline structure. SEM images of 92-nm-diameter (D) and 185-nm-diameter (E) pentahedrally twinned Au NWs. A 3D schematic representation (F) of the pentahedrally twinned $A u$ NWs.

simplifying purification and removal of excess CTAB. The Au NWs were purified further by centrifugation prior to deposition onto a substrate for electrical characterization.

This process allowed us to obtain Au NWs satisfying four conditions, i.e., they i) have a high aspect ratio (above 80), ii) are highly uniform (Fig. 1B), iii) are highly crystalline with a pentagonal cross-section (Fig. 1C-F), ${ }^{[33]}$ and iv) have sufficiently small diameter to carry out the meaningful measurements of electrical transport representative of nanoscale metals. Note that the crystal structure of the NWs is well known, ${ }^{[33]}$ and the interfaces between the different crystals are oriented along the long NW axis, i.e., parallel to charge transport direction.

A multi-probe scanning tunneling microscope (MPSTM) equipped with a scanning electron microscope (SEM) (Nanoprobe, Omicron) was used to image and to measure the transport properties of the Au NWs because it allows one to make several measurements over the length of the NW, and select the contact points. ${ }^{[29]}$ MPSTM transport measurements require long and sharp STM tips to achieve close proximately (Fig. 2A). The tungsten tips for this study were prepared by electrochemical etching under a magnetic field. ${ }^{[36]}$ The $I-V$ curve (Fig. 2B) acquired for the exact configuration shown in Figure 2A indicates
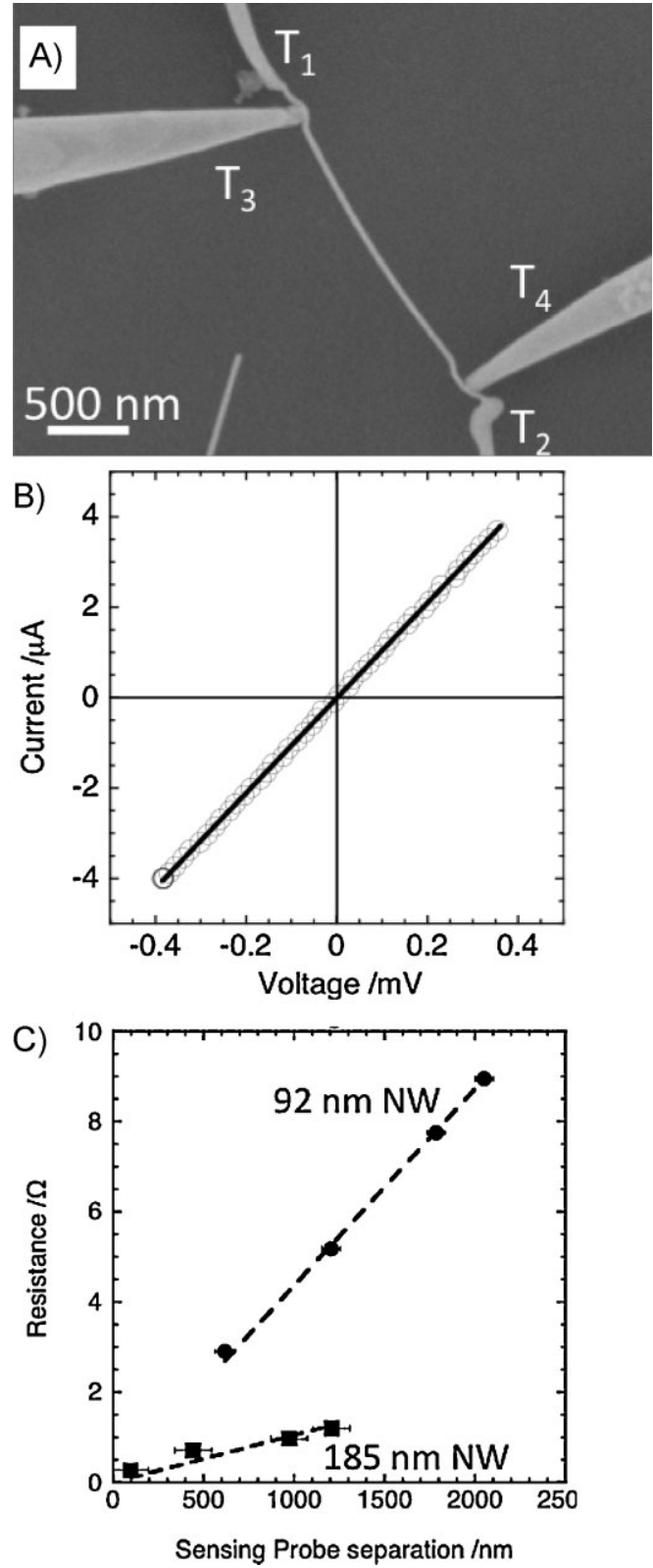

Figure 2. A) SEM image of four independent tungsten STM tips ( $T 1, T 2$, T3, and T4) making contact with the 29-nm diameter Au NW. The STM tips entering from the top (T1) and the bottom (T2) of the image were used to source current. The tips entering the image from the left (T3) and right (T4) were used to sense the voltage. B) The $I-V$ plot of a four-probe measurement on a 29-nm diameter Au NW with a line of best fit. C) Resistance versus sensing probe separation of the $92-\mathrm{nm}$ and $185-\mathrm{nm}$ diameter $\mathrm{Au}$ NW.

that the 29-nm-diameter Au NWs behave in a perfectly Ohmic manner. The resistance of the $29 \mathrm{~nm} \mathrm{NW}$ between the sensing tips, Figure 2, was found to be $95.2 \pm 0.1 \Omega$. The resistivity of this NW can be calculated by measuring the cross-sectional area and the length of this individual NW measured to be $1975 \pm 25 \mathrm{~nm}$. Given the mean diameter of the NW is $29 \mathrm{~nm}$ and that the cross-sectional is pentagonal (Fig. 1), the Au NW resistivity, $\rho_{\mathrm{NW}}$, 


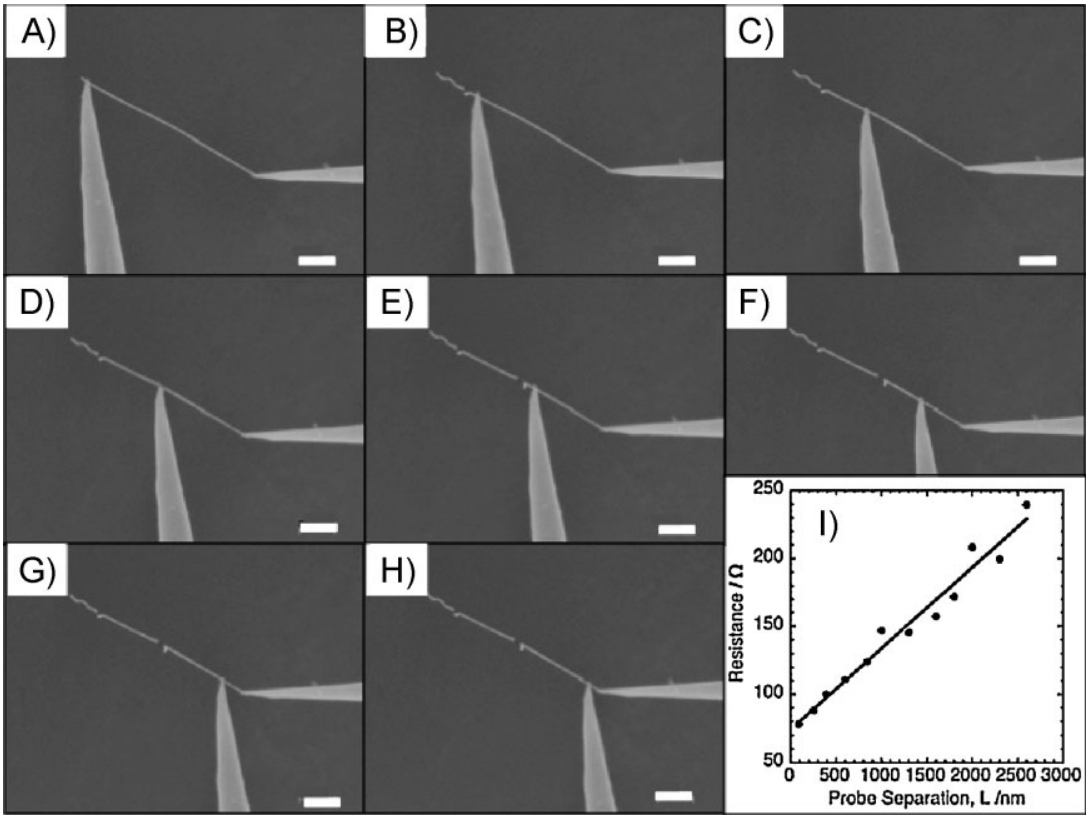

Figure 3. A chronological series $(A-H)$ of SEM images of a single NW $(29 \mathrm{~nm})$ being probed by tungsten STM tips. The STM tip on the right remained stationary and in contact with the NW while the STM tip entering from the bottom of each image was moved along the Au NW systematically. I) The plot of resistance versus separation for the Au NW. The scale bars are $500 \mathrm{~nm}$.

is estimated to be $2.50 \pm 0.09 \mu \Omega . \mathrm{cm}$. The room temperature bulk resistivity of gold is quoted to be and $2.2 \mu \Omega . \mathrm{cm} .{ }^{[37,38]}$ Therefore, the resistivity of gold NWs in this study was approximately 1.14 times that of the bulk metal. Given that these NWs are just $29 \mathrm{~nm}$ in diameter the resistivity is very low, especially when one considers the broad range of resistivities quoted in the literature. ${ }^{[27,28]}$ One of the advantages of the MPSTM technique is the possibility of plotting resistance against sensing tip separation, allowing one to calculate the resistivity of NWs using several measurements. ${ }^{[2,29,36]}$ Resistance versus probe separation data for the 92-nm and 185-nm diameter Au NWs are shown in Figure $2 \mathrm{C}$, which correspond to resistivities of $2.19 \pm 0.06 \mu \Omega . \mathrm{cm}$ and $2.1 \pm 0.2 \mu \Omega . \mathrm{cm}$, respectively, i.e. corresponding to bulk gold.

The 29-nm-diameter NWs are thinner and more ductile, which makes contacting the $\mathrm{Au} \mathrm{NW}$ with four tips at different points along the NW more demanding. Thin Au NWs tend to bend and can be damaged during the contact with the STM tips making multiple separation four-probe measurements difficult. For this reason, we have also adopted the two-probe approach to measure the resistance as a function of separation along $29 \mathrm{~nm} \mathrm{Au} \mathrm{NWs.}$ Figure $3(\mathrm{~A}-\mathrm{H})$ shows a single Au NW being probed by two STM tips. One of the STM tips is moved, while the other is fixed to reduce errors in separation. The first measurement (Fig. 3A) is made at the largest separation so that any damage made during contact to the $\mathrm{Au} \mathrm{NW}$ has a minimal effect on subsequent measurements. The plot of resistance versus separation is shown in Figure 3I. The gradient of the line of best fit, $\Delta R / \Delta L$, was $5.96 \pm 0.35 \times 10^{7} \Omega \mathrm{m}^{-1}$ and the intercept gives the average contact resistance to be $74 \pm 5 \Omega$. The resistivity of the NW gives the resistivity, $\rho_{\mathrm{NW}}$, was determined to be $3.1 \pm 0.2 \mu \Omega \mathrm{cm}$, which is expectedly higher than that determined by the four probe technique.

Grain boundary reflections in thin films and wires have been studied both experimentally and theoretically. ${ }^{[6,39-44]}$ Overall, it was concluded that grain boundary reflections are the dominant contribution to the resistivity of NWs (Supporting Information). ${ }^{[6,40]}$

Here, we have fabricated highly crystalline NWs that will have a negligible contribution from grain boundary effects, and we therefore only need to consider the effects of surface scattering. Resistance caused by surface scattering is expected to become increasingly significant as the diameter of the NW approaches and drops below the bulk mean free path. A common approach to understanding conductivity of nanoscale materials is to use the Fuchs approximation. ${ }^{[45]}$ However, it is only valid for cases when the thickness, or diameter, of the material is much greater than the mean free path and is therefore not appropriate for the NWs in this study. A more relevant model for circular cross-sectional wires was reported by Dingle (see Supporting Information), where the scattering was assumed to be inelastic. ${ }^{[46]}$

Dingle's modeled resistivity versus diameter is plotted in Figure 4A. However, the NWs fabricated in our study have a pentagonal cross-section, and therefore due to geometry, one might expect the degree of scattering to differ. We therefore derived an expression for the resistivity of pentagonal NWs based on the work of Dingle and Chambers (Supporting Information Equation S3). ${ }^{[4,47]}$ The model assumes that the surface scattering is inelastic, thereby giving the maximum expected resistivity of gold for a given diameter (Fig. 4A). The resistivities of Au NWs reported before are nearly all much higher than predicted by surface scattering alone (Fig. 4A). It is highly likely that the combination of grain boundary reflections and surface scattering lead to these reportedly large resistivities since nearly all of the NWs reported are polycrystalline. Importantly, the resistivity values of the pentahedrally twinned $\mathrm{Au} \mathrm{NWs}$ in this study are much lower than predicted by this model (Fig. 4A), thus we must conclude that the NW do not purely scatter inelasticity, but must also "reflect" electrons at the surfaces. ${ }^{[46,48]}$ Using the Sambles approach, we can derive the equation for NW, which implies that the resistivity is dependent on surface roughness, radius, and mean free path (Supporting Information Equation S4) and plot the resistivity as a function of diameter and the roughness parameter, $H$. As expected the resistivity of gold increases with decreasing diameter (Fig. 4B). However, as the NW surfaces become smoother $(H<1)$ the resistivity tends towards the bulk value. In the other limiting case, when $H>>1$, the surface of the wire scatters almost all of the incident electrons and tends towards Dingles inelastic scattering model (Fig 4A). It should be noted that the resistivity of highly scattering cylindrical NWs (Dingle's model) closely follows the resistivity expected from inelastical scattering pentagonal cross-section NWs (Supporting 

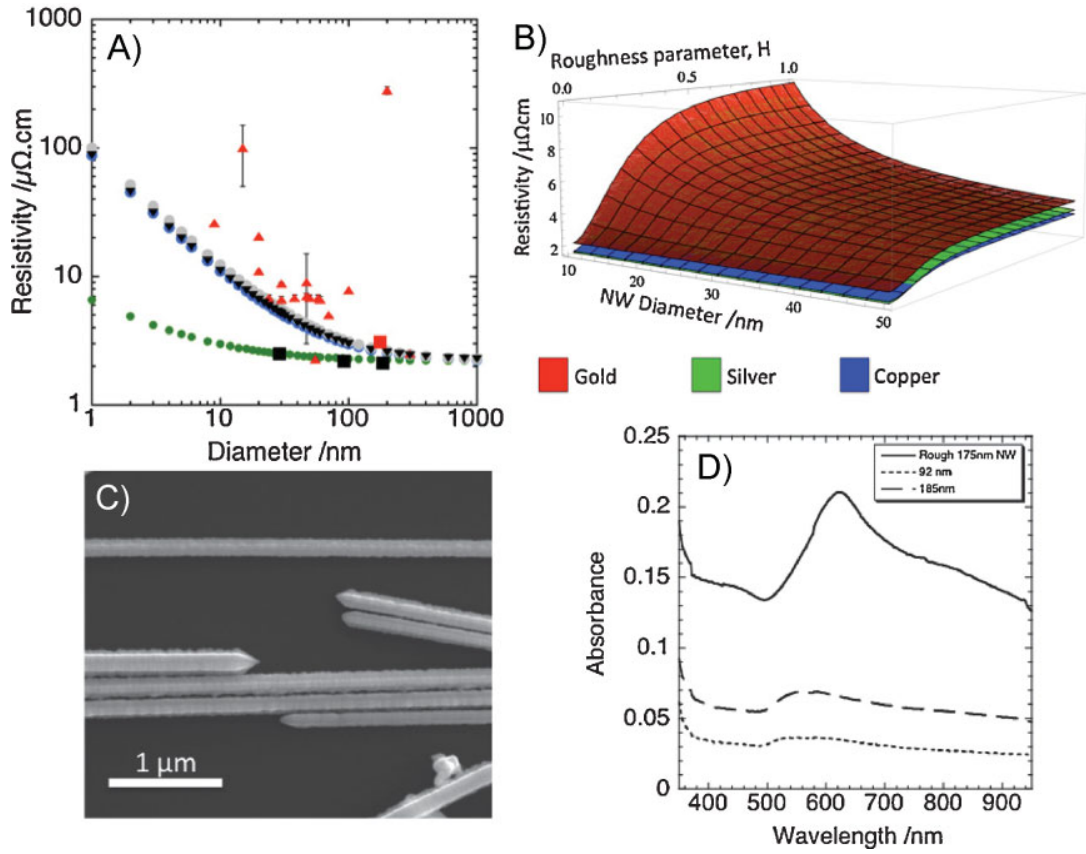

Figure 4. The resistivity of gold nanowires versus diameter. A) The resistivities of the smooth $A u$ NWs measured in this study are represented by the black squares. The resistivity of a NW with a rough surface is given by the red square. Dingle's model (Supporting Information) of the resistivity of cylindrical wires is represented by black triangles. The resistivity of wires with a pentagonal cross-section, (Equation S3, Supporting Information) are represented by grey circles. The blue circles represent the resistivity of cylindrical gold NWs modeled using Equation 2 with $\mathrm{H}$ $>>1$ (inelastical scattering). The green circles represent the modeled resistivity of atomically smooth cylindrical NWs (with $H=0.055)$ using (Equation S4, Supporting Information). Experimentally determined resistivities of $\mathrm{Au} N \mathrm{NW}$ reported in the literature are represented by red triangles (see Table S1 in Supporting Information). B) A 3D plot showing the resistivity of Au, Ag, and $\mathrm{Cu}$ over a range of roughness parameters, $\mathrm{H}$, and wire diameters (Equation S4, Supporting Information). C) A SEM image of the NWs with roughened surface. D) UV-vis spectrum of the 92-nm (dotted line), 185-nm (dashed line), and 176-nm diameter rough surface (solid line) NW dispersions in water.
Atomically smooth surfaces are 'mirror-like' to electrons and reflect a large portion ( 90\%) of the incident electrons, thereby reducing the surface scattering effect to keep the resistivity close to bulk (Fig. 4B). Importantly, these findings are quite general and similar dependences were found for other metals typically used in electronics, such as $\mathrm{Ag}$ and $\mathrm{Cu}$ (Fig. 4B). A recent article demonstrated that the longitudinal surface plasmon is sensitive to atomic surface roughness in nanorods. ${ }^{[51]}$ UV-vis data of the 92-nm, 185-nm, and the 176-nm (rough) NWs are shown in Figure 4D. In agreement with theoretical work of Pecharromán et al. we find that the "rough" NW surface plasmon peak is red shifted (in this case $75 \mathrm{~nm}$ ) with respect to the smooth NW peaks. ${ }^{[51]}$ This is a further indication that surface plasmon data for gold NWs contains information about the atomic roughness of the surface, which has a direct impact on the resistivity of the NW.

These data indicate that NWs can possess near-bulk conductivity if the NWs are highly crystalline (few or preferably no grain boundaries) and are highly smooth. Low resistance nanoscale interconnects and elimination of grain boundaries perpendicular to carrier transport are important for future nanocircuits from the perspective of computational performance, device noise, and energy consumption. We have established in this study that it is fundamentally possible to make nanoscale interconnects with near-bulk conductivity, which is much lower than the current ones made by photolithography.
Information, Equation S3) and therefore, the derived equation can be used as a good approximation for the NWs.

Experimentally determined NW resistivity values can be fitted to our model, by varying the roughness parameter $H$. If the mean free path of the conducting electrons is taken to be $38 \mathrm{~nm}$ for gold $^{[43]}$ then a good fit could be achieved when $H$ is set to a value of $\sim 0.055$ (Fig. 4A). Given that $H=h / \lambda_{F}$, where $h$ is the RMS roughness and $\lambda_{F}$ is the electron wavelength (taken to be $\sim 5 \AA^{[38]}$ ) this would correspond to a NW RMS roughness of $\sim 0.3 \AA$ i.e. atomically smooth NWs ${ }^{[49]}$. Furthermore, our data fits well with NWs with a $55 \mathrm{~nm}$ diameter recently reported elsewhere. ${ }^{[50]}$ In order to confirm our findings we choose to measure the resistivity of a NW with a rough surface (Fig. 4C). Four-probe STM measurements on the rough NW gave a resistance per length of $1.64 \pm 0.02 \Omega \mu \mathrm{m}^{-1}$. The cross-sectional area of the rough NWs is calculated to be $1.89 \times 10^{-14} \mathrm{~m}^{2}$ giving a resistivity of $3.1 \pm 0.3 \Omega \mathrm{cm}$. Figure $4 \mathrm{~A}$ shows that the resistivity of the rough NW is within experimental error of the theoretically determined 'purely' scattering surface $(\mathrm{H}>>1)$ and is certainly much higher than the smooth NWs, which confirms that surface roughness has the effect of increasing the resistivity.

\section{Experimental}

All experimental details are given in the Supporting Information.

\section{Acknowledgements}

NAK thanks NSF, AFOSR, ONR, and DARPA for financial support of this research. KC and SDE thanks Marie Curie Actions MOIF-CT-2006-039636 for financial support. MG thanks the University of Leeds for the provision of a PhD scholarship. Support for ERZ was provided by the Robert A. Welch Foundation (C-1703), NSF (DMR-0547399 and CBET-0506832), and Alliance for Nanohealth (W8XWH-07-2-0101). Supporting Information is available online from Wiley InterScience or from the author.

Received: January 21, 2010 Published online: April 7, 2010

[1] K. Kordás, G. Tóth, P. Moilanen, M. Kumpumäki, J. Vähäkangas, A. Uusimäki, Appl. Phys. Lett. 2007, 90, 123105.

[2] J. D. Meindl, IEEE Micro. 2003, 23, 28. 
[3] M. Forshaw, R. Stadler, D. Crawley, K. Nikolic, Nanotechnology 2004, 15, S220.

[4] J. M. Rabaey, A. Chandrakasan, B. Nikolic, Digital Integrated Circuits, Prentice Hall, 2003.

[5] R. Lin, P. Bøggild, O. Hansen, J. Appl. Phys. 2004, 96, 2895.

[6] A. Bietsch, B. Michel, Appl. Phys. Lett. 2002, 80, 3346.

[7] M. Ou, S. Harutyunyan, S. Lai, C. Chen, T. Yang, Y. Chen, Phys. Status Solidi B 2007, 244, 4512.

[8] E. Menke, M. Thompson, C. Xiang, L. Yang, R. Penner, Nat. Mater. 2006, 5, 914.

[9] T. Bhuvana, G. U. Kulkarni, ACS Nano 2008, 2, 457.

[10] G. Marzi, D. Iacopino, A. Quinn, G. Redmond, J. Appl. Phys. 2004, 96, 3458.

[11] W. Gu, H. Choi, K. K. Kim, Appl. Phys. Lett. 2006, 89, 253102.

[12] S. Valizadeh, M. Abid, F. Hernandez-Ramirez, A. R. Rodriguez, K. Hjort, J. A. Schweitz, Nanotechnology 2006, 17, 1134.

[13] J. Ahn, G. Ko, J. Kim, M. Mastro, J. Hite, C. Eddy, Curr. Appl. Phys. 2010, 10, 703.

[14] Y. G. Li, Y. Y. Wu, J. Am. Chem. Soc. 2009, 131, 5851.

[15] J. J. Boote, S. D. Evans, Nanotechnology 2005, 16, 1500.

[16] S. Jin, D. Whan, M. C. McAlpine, R. S. Friedman, Y. Wu, C. M. Lieber, Nano Lett. 2004, 4, 915

[17] Y.-K. Kim, P. S. Kang, D. Kim, G. Shin, G. T. Kim, J. S. Ha, Small 2009, 5, 727.

[18] Y. Yadav, S. K. Padigi, S. Prasad, X. Y. Song, J. Nanosci. Nanotech. 2008, 8, 1951.

[19] Z. Y. Fan, J. C. Ho, Z. A. Jacobson, R. Yerushalmi, R. L. Alley, H. Razavi, A. Javey, Nano Lett. 2008, 8, 20.

[20] J. Boote, K. Critchley, S. Evans, in J. of Expt. Nanoscience, Vol. 1, 2006, 125.

[21] S. Hong, T. H. Kim, J. Lee, K. E. Byun, J. Koh, T. Kim, S. Myung, Nano 2007, 2, 333.

[22] K. Keshoju, H. Xing, L. Sun, Appl. Phys. Lett. 2007, 91, 123114.

[23] Y. Huang, X. F. Duan, Q. Q. Wei, C. M. Lieber, Science 2001, 291, 630.

[24] S. Koh, Nanoscale Research Lett. 2007, 2, 519.

[25] K. Biswas, Y. Qin, M. Dasilva, R. Reifenberger, T. Sands, Phys. Status Solidi A 2007, 204, 3152.

[26] M. L. Gorzny, A. S. Walton, M. Wnek, P. G. Stockley, S. Evans, Nanotechnology 2008, 19, 165704.
[27] J. H. Song, Y. Y. Wu, B. Messer, H. Kind, P. D. Yang, J. Am. Chem. Soc. 2001, 123, 10397.

[28] C. Wang, Y. Hu, C. M. Lieber, S. Sun, J. Am. Chem. Soc 2008, 130, 8902.

[29] A. S. Walton, C. S. Allen, K. Critchley, M. L. Gorzny, J. E. McKendry, R. M. D. Brydson, B. J. Hickey, S. Evans, Nanotechnology 2007, 18, 065204.

[30] B. D. Busbee, S. O. Obare, C. J. Murphy, Adv. Mater. 2003, 15, 414

[31] S. M. Marinakos, S. Chen, A. Chilkoti, Anal. Chem. 2007, 79, 5278.

[32] A. K. Oyelere, P. C. Chen, X. Huang, I. H. El-Sayed, M. A. El-Sayed, Bioconjugate Chem. 2007, 18, 1490.

[33] C. J. Johnson, E. Dujardin, S. A. Davis, C. J. Murphy, S. Mann, J. Mater. Chem. 2002, 12, 1765.

[34] C. R. Martin, Science 1994, 266, 1961.

[35] B. Wu, J. J. Boland, J. Colloid Interface Sci. 2006, 303, 611.

[36] J. E. McKendry, C. S. Allen, K. Critchley, M. L. Gorzny, A. S. Walton, S. Evans, Nanotechnology 2008, 19, 085201.

[37] R. C. Munoz, G. Vidal, M. Mulsow, J. G. Lisoni, C. Arenas, A. Concha, F. Mora, R. Espejo, Phys. Rev. B 2000, 62, 4686

[38] J. R. Sambles, K. C. Elsom, D. J. Jarvis, Philos. Trans. R. Soc. London, A 1982, 304, 365.

[39] S. Karim, W. Ensinger, The Nucleus 2008, 45, 1.

[40] W. Steinhogl, G. Steinlesberger, M. Perrin, G. Scheinbacher, G. Schindler, M. Traving, M. Engelhardt, Microelectron. Eng. 2005, 82, 266.

[41] A. F. Mayadas, M. Shatzkes, Phys. Rev. B 1970, 1, 1382.

[42] R. Dimmich, F. Warkusz, Active and Passive Electron. Comp. 1986, 12, 103.

[43] Q. Zhang, B. Cao, X. Zhang, K. Takahashi, Phys. Rev. B 2006, 74, 5.

[44] W. Zhang, S. Brongersma, O. Richard, B. Brijs, R. Palmans, L. Froyen, K. Maex, Microelectron. Eng. 2004, 76, 146.

[45] K. Fuchs, Proc. Cambridge Philos. Soc. 1938, 34, 100.

[46] R. B. Dingle, Philos. R. Soc. London, Ser. A 1950, 201, 545.

[47] R. G. Chambers, Proc. R. Soc. of London. Series A. 1950, 378.

[48] S. B. Soffer, J. Appl. Phys. 1967, 38, 1710.

[49] J. R. Sambles, K. C. Elsom, T. W. Preist, J. Phys. F, Met. Phys. 1982, 12, 1169.

[50] Y. Peng, T. Cullis, B. Inkson, Appl. Phys. Lett. 2008, 93, 183112.

[51] C. Pecharroman, J. Perez-Juste, G. Mata-Osoro, L. Liz-Marzan, P. Mulvaney, Phys. Rev. B 2008, 77, 035418 